Acta Crystallographica Section C

Crystal Structure

Communications

ISSN 0108-2701

\section{Tosyl esters of cinchonidine and cinchonine alkaloids: the structure- reactivity relationship in the hydrolysis to 9-epibases}

\section{Zbigniew Karczmarzyk,* Teodozja M. Lipińska, Waldemar Wysocki, Monika Denisiuk and Katarzyna Piechocka}

Department of Chemistry, University of Podlasie, ul. 3 Maja 54, 08-110 Siedlce, Poland

Correspondence e-mail: kar@uph.edu.pl

Received 20 April 2011

Accepted 7 July 2011

Online 5 August 2011

In the crystal structures of the diastereoisomers of $O$-tosylcinchonidine [(9R)-cinchon-9-yl 4-methylbenzenesulfonate], (I), and $O$-tosylcinchonine [(9S)-cinchon-9-yl 4-methylbenzenesulfonate], (II), both $\mathrm{C}_{26} \mathrm{H}_{28} \mathrm{~N}_{2} \mathrm{O}_{3} \mathrm{~S}$, both molecules are in an anti-closed conformation and, in each case, the position of the aryl ring of the tosylate system is influenced by an intramolecular $\mathrm{C}-\mathrm{H} \cdots \mathrm{O}$ hydrogen bond. The molecular packing in (I) is influenced by weak intermolecular $\mathrm{C}-\mathrm{H} \cdots \mathrm{O}$ and $\mathrm{C}-\mathrm{H} \cdots \pi$ interactions. The crystal structure of (II) features $\mathrm{C}-\mathrm{H} \cdots \pi$ interactions and van der Waals forces only. The computational investigations using RHF/6-31G** $a b$ initio and AM1 semi-empirical methods performed for (I) and (II) and their protonated species show that the conformational and energetic parameters of the molecules are correlated with differences in their reactivity in hydrolysis to the corresponding 9-epibases.

\section{Comment}

Studies on the difference in biological activity of natural Cinchona alkaloids with respect to their structural, stereochemical and physicochemical properties have attracted much attention owing to the pharmacological interest in these compounds (Verpoorte et al., 1988). Recently, Cinchona alkaloids and their derivatives have been investigated as natural organocatalysts giving asymmetric induction in organic reactions with the formation of stereogenic centres (Song, 2009). Transformation of natural alkaloids into pharmacologically inactive 9-epibases is known to be a two-step process: formation of sulfonate esters followed by hydrolysis in a weak acid medium (Hoffman \& Frackenpohl, 2004). The first step proceeds with retention and the second one with inversion of the carbinol atom configuration. It was found that hydrolysis of $O$-tosyl derivatives is a good method for epimerization of C9 in the quinine, quinidine and cinchonidine cores, but is ineffective for cinchonine since its tosylate converts slowly and not selectively to the corresponding 9-epibase (Braje et al., 2000). In order to link the differences in experimental reactivity in the hydrolysis to 9-epibases with structural and energetic parameters, $\mathrm{X}$-ray investigations and theoretical calculations were undertaken using cinchonidine and cinchonine tosylates, (I) and (II), as model compounds.

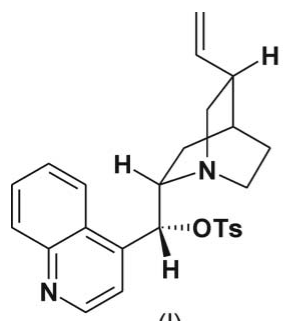

(I)

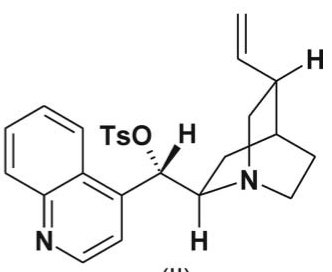

(II)
Structural analysis of the diastereoisomeric molecules (I) and (II) confirms the retention of the original, respective, $R$ and $S$ configurations at atom $C 9$ in the crystals of both tosylates (Figs. 1 and 2, respectively). The geometry (bond lengths, angles and planarity) of the main Cinchona alkaloid skeleton is similar in (I) and (II) and the related parent structures of cinchonidine and cinchone molecules (Oleksyn, 1982; Oleksyn et al., 1979). Both molecules adopt an anti-closed conformation, torsion angles $\varphi_{1}=\mathrm{N} 1-\mathrm{C} 8-\mathrm{C} 9-\mathrm{O} 1=166.6(6)$ and $-176.7(4)^{\circ}, \varphi_{2}=\mathrm{N} 1-\mathrm{C} 8-\mathrm{C} 9-\mathrm{C} 24=48.4(6)$ and $-57.6(6)^{\circ}$,

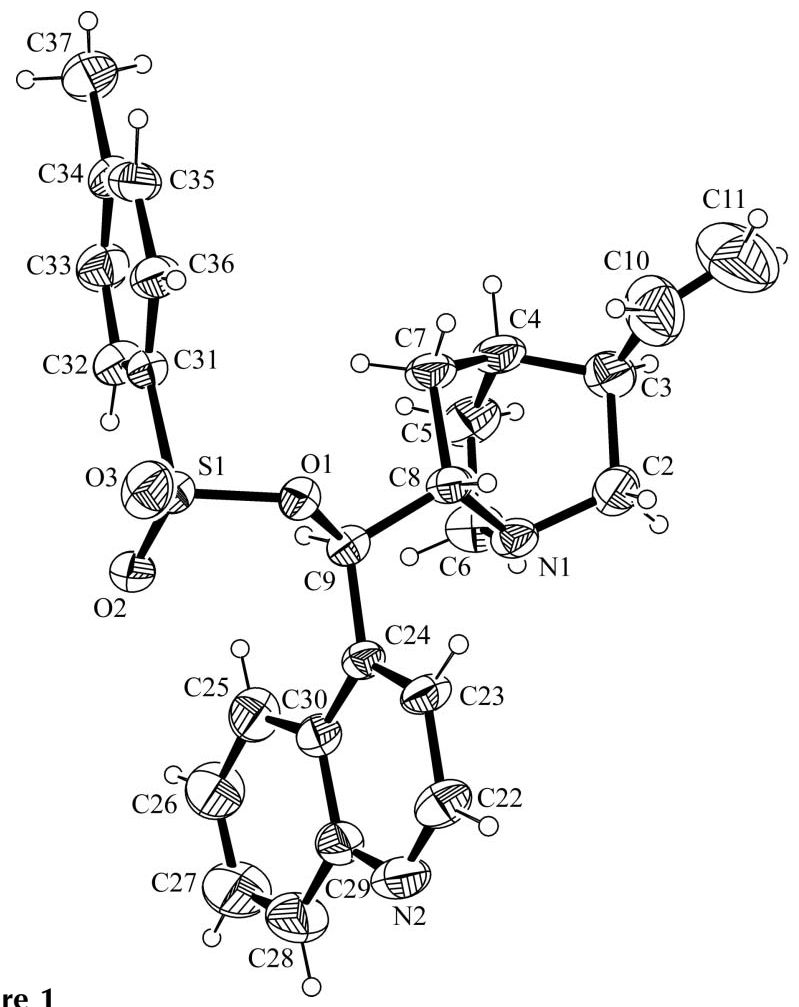

A view of (I) showing the atom-numbering scheme. Displacement ellipsoids are drawn at the $30 \%$ probability level. $\mathrm{H}$ atoms are represented as small spheres of arbitrary radii. 
$\varphi_{3}=\mathrm{O} 1-\mathrm{C} 9-\mathrm{C} 24-\mathrm{C} 23=-59.7(4)$ and $50.9(6)^{\circ}$, and $\varphi_{4}=$ $\mathrm{C} 8-\mathrm{C} 9-\mathrm{C} 24-\mathrm{C} 23=55.9(5)$ and $-65.7(6)^{\circ}$ in (I) and (II), respectively, which is characteristic, for example, for $O$-mesylquinidine (Braje et al., 2000) and is in contrast to an anti-open conformation observed for the parent alkaloids. The four conformers, viz. anti-closed, syn-closed, anti-open and synopen (Caner et al., 2003), of the cinchonine-type molecule, showing the lowest energy, are presented in Fig. 3.

The orientation of the vinyl substituent in relation to the quinuclidine system is different in (I) and (II): the torsion angle $\mathrm{C} 2-\mathrm{C} 3-\mathrm{C} 10-\mathrm{C} 11$ describing this orientation is $105.4(13)^{\circ}$ in (I) and $175.0(12)^{\circ}$ in (II). The gauche conformation of the vinyl group in (I) may be caused by a weak intermolecular $\mathrm{C} 10-\mathrm{H} 101 \cdots \mathrm{O} 3$ hydrogen bond and a $\mathrm{C} 11-$ H111 $\cdots \pi$ interaction (Table 1). Similarly, the trans conformation of the vinyl group in (II) may be a result of the weak $\mathrm{C} 11-\mathrm{H} 111 \cdots \pi$ (quinoline) intermolecular interaction (Table 2). The aryl ring of the tosyl group is inclined to the quinoline ring at angles of 20.24 (10) and 11.51 (13) in (I) and (II), respectively, and its position is influenced by the C32$\mathrm{H} 321 \cdots \mathrm{O} 2$ short intramolecular contact (Tables 1 and 2).

The hydrolysis of $O$-tosylated molecules proceeds with inversion of the $\mathrm{C} 9$ configuration as an $\mathrm{S}_{\mathrm{N}} 2$ attack by the nucleophilic water molecule from the opposite site to the tosylate leaving group in the substrate requires it to be protonated at the quinuclidine $\mathrm{N}$ atom. This process is favoured when the substrate molecule can change from an

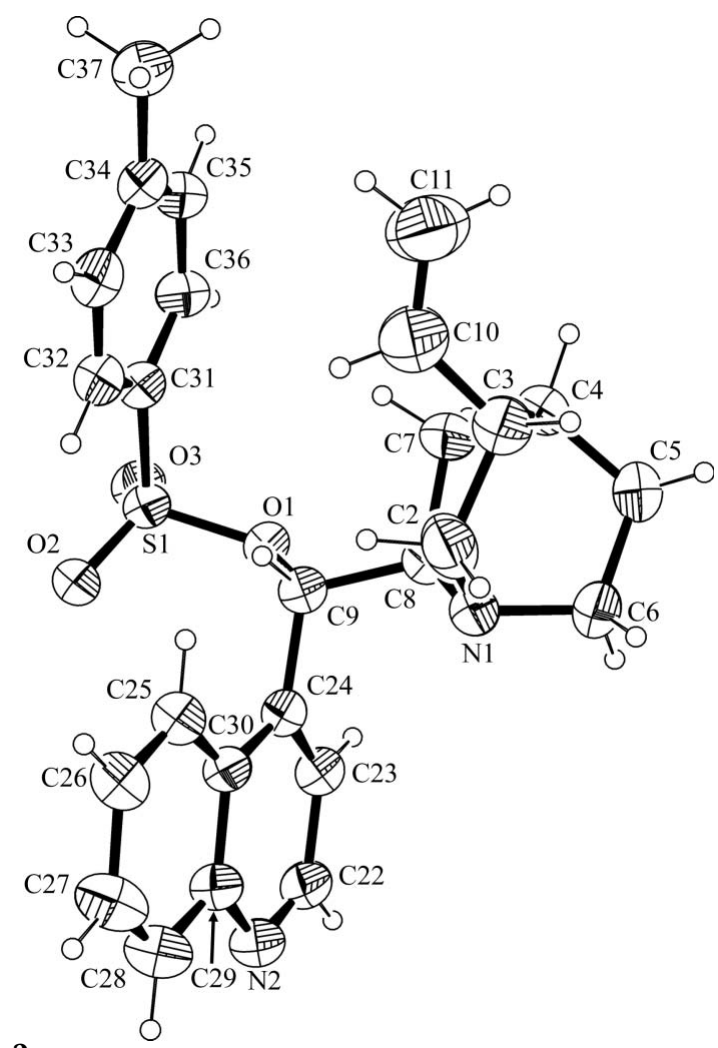

Figure 2

A view of (II) showing the atom-numbering scheme. Displacement ellipsoids are drawn at the $30 \%$ probability level. $\mathrm{H}$ atoms are represented as small spheres of arbitrary radii. anti-closed conformation, observed in the crystal, into a synopen one which is optimal for the $\mathrm{S}_{\mathrm{N}} 2$ attack in the aqueous weak acid medium. It can be assumed on the basis of known reactivity that the transition state may be formed more easily in the case of cinchonidine tosylate (I) than in the case of cinchonine tosylate (II). The theoretical calculations at the RHF SCF ab initio 6-31G** level (Bylaska et al., 2006; Kendall et al., 2000) show that the conformations of molecules (I) and (II) as observed in their crystals are not equi-energetic, with a difference in energy between the (I) and (II) conformations of $\Delta E=2.57 \mathrm{kcal} \mathrm{mol}^{-1}\left(1 \mathrm{kcal} \mathrm{mol}^{-1}=\right.$ $4.184 \mathrm{~kJ} \mathrm{~mol}^{-1}$; single-point energy calculations). The energy minimization and full geometry optimization with initial geometries obtained from the X-ray analysis for molecules (I) and (II) yielded a smaller difference in energy of $0.85 \mathrm{kcal} \mathrm{mol}^{-1}$ between the conformations of molecule (I) $\left(\varphi_{1}=-178.8^{\circ}, \varphi_{2}=62.8^{\circ}, \varphi_{3}=-44.0^{\circ}\right.$ and $\left.\varphi_{4}=72.1^{\circ}\right)$ and (II) $\left(\varphi_{1}=174.9^{\circ}, \varphi_{2}=-66.6^{\circ}, \varphi_{3}=39.8^{\circ}\right.$ and $\left.\varphi_{4}=-76.8^{\circ}\right)$ than that reported for the single-point calculation. It is clear that these energy values do not prevent molecule (II) from changing from an unfavourable anti-closed conformation to a syn-open conformation as expected in the $\mathrm{S}_{\mathrm{N}} 2$ hydrolysis reaction. The calculations performed for N1-protonated molecules in the syn-open conformation after energy minimization and geometry optimization $\left[\varphi_{1}=-54.0\right.$ and $48.9^{\circ}, \varphi_{2}=179.3$ and $176.4^{\circ}, \varphi_{3}=146.8$ and $-144.6^{\circ}$, and $\varphi_{4}=-87.2$ and $88.5^{\circ}$ for (I) and (II), respectively] gave a difference in energy between the protonated (II) and (I) species of $0.39 \mathrm{kcal} \mathrm{mol}^{-1}$ and, moreover, a larger energetic profit of $1.84 \mathrm{kcal} \mathrm{mol}^{-1}$ after protonation of (I) compared with (II) with respect to the free $O$ tosylates in an anti-closed conformation. Therefore, the different reactivity of (I) and (II) towards the appropriate 9-epibases may be related to a change in energy during protonation on the $\mathrm{N} 1$ atom and a change in conformation from anti-closed to $s y n$-open during the hydrolysis process. In
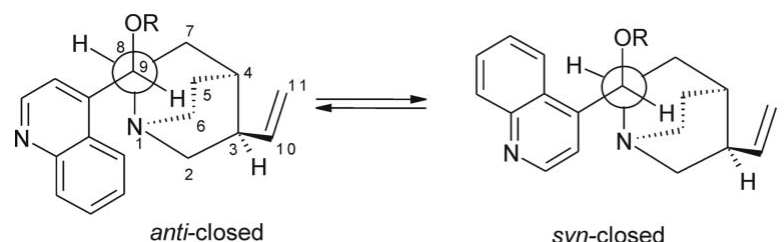

anti-closed

$$
\text { syn-closed }
$$
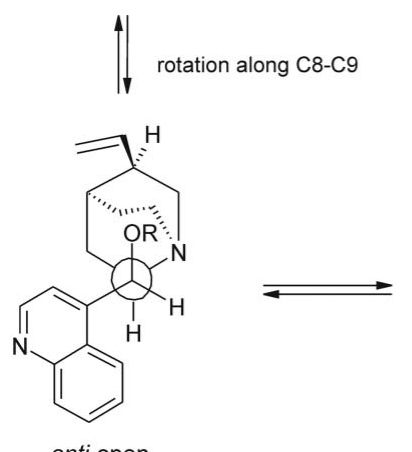

anti-open

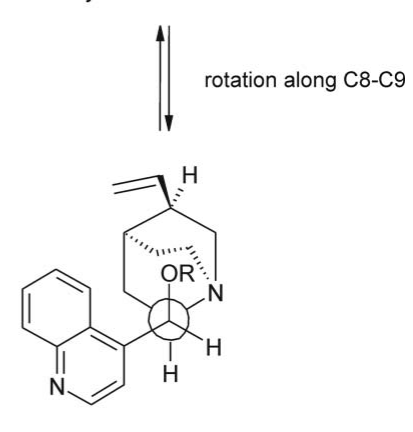

syn-open

\section{Figure 3}

The four conformers of cinchonine showing the lowest energies. 
order to confirm this conclusion, the hydrolysis process was modelled using the N1-protonated molecules of (I) and (II) in 'crystallographic' anti-closed conformation and an anion of salicylic acid in a water environment (as an aqueous weak acid medium). The water environment was simulated by locating the alkaloid and salicylate ion in the centre of the box surrounded by 17 water molecules equilibrated at $300 \mathrm{~K}$ and $1013 \mathrm{hPa}$ (Jorgensen et al., 1983). The energy minimization and geometry optimization of the (I)- $\mathrm{H}^{+}$-salicylate ${ }^{-}-\mathrm{H}_{2} \mathrm{O}$ system using the semi-empirical AM1 method implemented in the HYPERCHEM package (Hypercube, 1998) give molecule (I) an anti-open conformation $\left(\varphi_{1}=-63.7^{\circ}, \varphi_{2}=175.4^{\circ}, \varphi_{3}=\right.$ $-17.1^{\circ}$ and $\left.\varphi_{4}=102.3^{\circ}\right)$ which is closely related by rotation

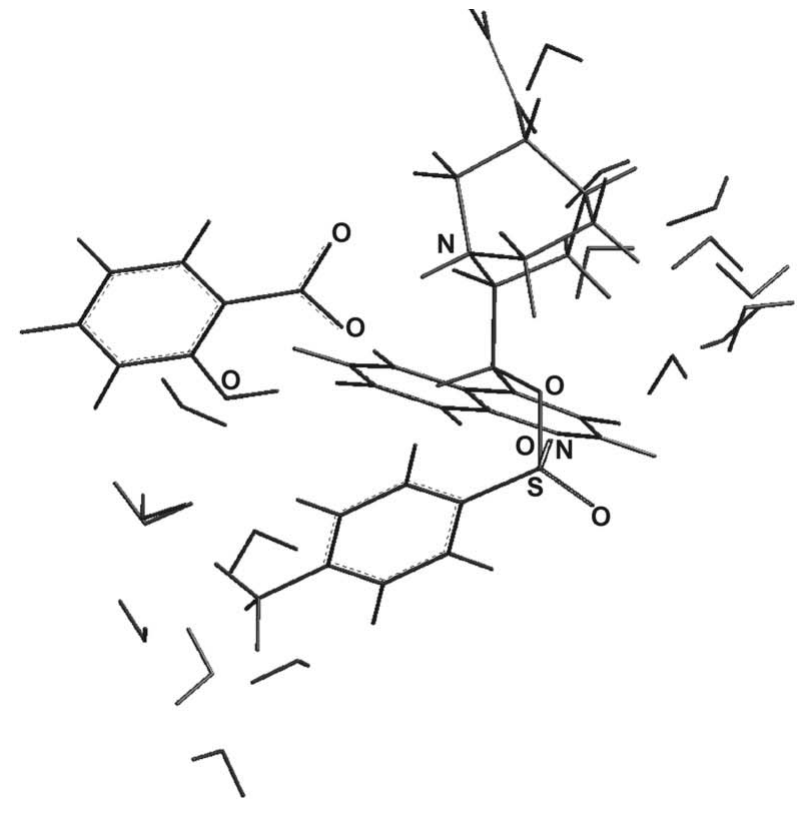

(a)

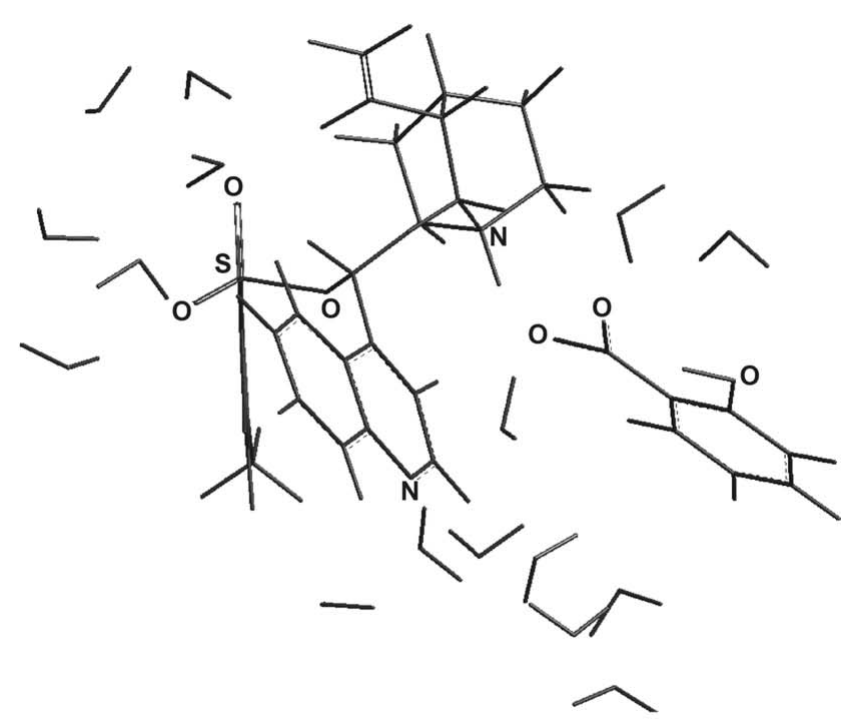

Figure 4

(b)

The optimized conformations of $(a)$ the (I)- $\mathrm{H}^{+}$-salicylate ${ }^{-}$and $(b)$ the (II) $-\mathrm{H}^{+}$-salicylate ${ }^{-}$system in a water environment using the AM1 method.

around the $\mathrm{C} 9-\mathrm{C} 24$ bond to the syn-open conformation preferred for 9-epicinchonidine formation in the hydrolysis reaction (Figs. 3 and $4 a$ ). The parallel calculation for the (II)$\mathrm{H}^{+}$-salicylate ${ }^{-}-\mathrm{H}_{2} \mathrm{O}$ system retains molecule (II) in an anticlosed conformation, unfavourable for the hydrolysis reaction $\left(\varphi_{1}=-146.0^{\circ}, \varphi_{2}=-28.5^{\circ}, \varphi_{3}=49.4^{\circ}\right.$ and $\left.\varphi_{4}=-65.4^{\circ}\right)$ as shown in Fig. 4(b). Additionally, the (I)- $\mathrm{H}^{+}$-salicylate ${ }^{-}$system in the gaseous phase with (I) in an anti-open conformation is more energetically stable than the (II)- $\mathrm{H}^{+}$-salicylate ${ }^{-}$system with (II) in an anti-closed conformation with a $\Delta E$ value of $8.062 \mathrm{kcal} \mathrm{mol}^{-1}$. As can be seen in Fig. 4, the steric hindrance of the aryl ring of the tosylate group and the quinoline ring can restrain the free rotation on the $\mathrm{C} 24-\mathrm{C} 9$ bond, making the $\mathrm{C} 9$ atom more accessible to nucleophilic attack by the water molecule in an anti-open conformation of (I)- $\mathrm{H}^{+}$in comparison with an anti-closed conformation of (II)- $\mathrm{H}^{+}$. The stabilizing influence of the tosylate group on the conformations of (I) $-\mathrm{H}^{+}$and (II) $-\mathrm{H}^{+}$can result in their different behaviour in the hydrolysis reaction and their higher hydrolytic stability in comparison to $O$-mesyl and $O$-acyl Cinchona alkaloid derivatives.

In conclusion, the X-ray analysis and theoretical calculations provided the geometric, conformational and energetic parameters of the diastereoisomeric molecules $O$-tosyl cinchonidine, (I), and $O$-tosyl cinchonine, (II), which were used to explain their different reactivity in the hydrolysis to the respective 9-epibases. It appears that the different energetic profit during protonation on the $\mathrm{N} 1$ atom and the different propensity to change from an anti-closed conformation in the crystal to a syn-open one favoured in the hydrolysis process can be correlated with the different reactivity of (I) and (II) towards 9-epibases in the $\mathrm{S}_{\mathrm{N}} 2$ hydrolysis process.

\section{Experimental}

Compounds (I) and (II) were obtained according to the method described by Kowalik et al. (1999). The analytical data (IR, ${ }^{1} \mathrm{H}$ NMR and ${ }^{13} \mathrm{C}$ NMR) are in good agreement with those found by Brunner \& Bügler (1997) for (I) and Kowalik et al. (1999) for (II). Crystals of both compounds suitable for X-ray diffraction analysis were grown by slow evaporation from diethyl ether-hexane $(1: 1 \mathrm{v} / \mathrm{v})$ solutions.

\section{Compound (I)}

Crystal data

$\mathrm{C}_{26} \mathrm{H}_{28} \mathrm{~N}_{2} \mathrm{O}_{3} \mathrm{~S}$

$M_{r}=448.56$

Orthorhombic, $P 2_{1} 2_{1} 2_{1}$

$a=9.4591(13) \AA$

$b=10.094(2) \AA$

$c=24.370(4) \AA$

\section{Data collection}

Kuma KM-4 four-circle diffractometer

Absorption correction: multi-scan (Blessing, 1995)

$T_{\min }=0.363, T_{\max }=0.748$

3726 measured reflections

$$
\begin{aligned}
& V=2326.9(7) \AA^{3} \\
& Z=4 \\
& \text { Cu K } \alpha \text { radiation } \\
& \mu=1.48 \mathrm{~mm}^{-1} \\
& T=293 \mathrm{~K} \\
& 0.45 \times 0.40 \times 0.10 \mathrm{~mm}
\end{aligned}
$$

\footnotetext{
3508 independent reflections 1717 reflections with $I>2 \sigma(I)$ $R_{\text {int }}=0.034$

2 standard reflections every 100 reflections intensity decay: $0.0 \%$
} 


\section{Refinement}

$R\left[F^{2}>2 \sigma\left(F^{2}\right)\right]=0.064$

$w R\left(F^{2}\right)=0.167$

$S=1.06$

3508 reflections

290 parameters

1 restraint

\section{Compound (II)}

Crystal data

$\mathrm{C}_{26} \mathrm{H}_{28} \mathrm{~N}_{2} \mathrm{O}_{3} \mathrm{~S}$

$M_{r}=448.56$

Orthorhombic, $P 2{ }_{1} 22_{1}$

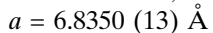

$b=17.7364(16) \AA$

$c=18.6632(17) \AA$

\section{Data collection}

Kuma KM-4 four-circle diffractometer

Absorption correction: $\psi$ scan

(North et al., 1968)

$T_{\min }=0.571, T_{\max }=0.847$

2806 measured reflections

\section{Refinement}

$R\left[F^{2}>2 \sigma\left(F^{2}\right)\right]=0.051$

$w R\left(F^{2}\right)=0.177$

$S=1.01$

2723 reflections

290 parameters

The assumed absolute stereochemistry of compound (I) was confirmed by refinement of the Flack (1983) parameter. In the absence of Friedel pairs, the absolute configuration of compound (II) was assigned from the absolute configuration of cinchonine as starting reagent in the stereoconservative synthesis. For both compounds, all $\mathrm{H}$ atoms were fixed geometrically and treated as riding on their parent $\mathrm{C}$ atoms, with $\mathrm{C}-\mathrm{H}$ distances of 0.93 (aromatic), $0.96\left(\mathrm{CH}_{3}\right), 0.97\left(\mathrm{CH}_{2}\right)$ and $0.98 \AA(\mathrm{CH})$, and with $U_{\text {iso }}(\mathrm{H})=1.5 U_{\text {eq }}(\mathrm{C})$. For both molecules, $\mathrm{C} 10$ and $\mathrm{C} 11$ of the terminal vinyl group showed large displacement parameters, which result in unrealistic $\mathrm{C} 10-\mathrm{C} 11$ bond lengths of 1.177 (8) and 1.206 (12) $\AA$ in (I) and (II), respectively. An electron-density map did not reveal the alternate sites for the $\mathrm{C} 10$ and $\mathrm{C} 11$ atoms. Therefore, a DFIX restraint (SHELXL97; Sheldrick, 2008) with a target value of 1.300 (5) $\AA$ for the $\mathrm{C} 10=\mathrm{C} 11$ vinyl bonds in (I) and (II) was used.

For both diastereoisomers, data collection: KM4B8 (Gałdecki et al., 1996); cell refinement: KM4B8; data reduction: DATAPROC (Gałdecki et al., 1995); program(s) used to solve structure: SIR92 (Altomare et al., 1993); program(s) used to refine structure: SHELXL97 (Sheldrick, 2008); molecular graphics: ORTEP-3 for Windows (Farrugia, 1997); software used to prepare material for publication: SHELXL97 and WinGX (Farrugia, 1999).

Supplementary data for this paper are available from the IUCr electronic archives (Reference: YF3003). Services for accessing these data are described at the back of the journal.
Table 1

Hydrogen-bond geometry $\left(\AA,^{\circ}\right)$ for (I).

$\operatorname{Cg} A, \operatorname{cg} B$ and $\operatorname{cg} C$ are the centroids of the benzene, toluene and pyridine rings, respectively.

\begin{tabular}{lllll}
\hline$D-\mathrm{H} \cdots A$ & $D-\mathrm{H}$ & $\mathrm{H} \cdots A$ & $D \cdots A$ & $D-\mathrm{H} \cdots A$ \\
\hline $\mathrm{C} 32-\mathrm{H} 321 \cdots \mathrm{O} 2$ & 0.93 & 2.60 & $2.944(9)$ & 103 \\
$\mathrm{C} 10-\mathrm{H} 101 \cdots \mathrm{O} 3^{\mathrm{i}}$ & 0.93 & 2.48 & $3.377(7)$ & 163 \\
$\mathrm{C} 11-\mathrm{H} 111 \cdots \mathrm{Cg} A^{\text {ii }}$ & 0.93 & 2.98 & $3.775(8)$ & 144 \\
$\mathrm{C} 23-\mathrm{H} 231 \cdots \mathrm{Cg} B^{\mathrm{i}}$ & 0.93 & 2.87 & $3.619(5)$ & 138 \\
$\mathrm{C} 37-\mathrm{H} 371 \cdots \mathrm{Cg} C^{\mathrm{iii}}$ & 0.96 & 2.81 & $3.746(7)$ & 165 \\
\hline
\end{tabular}

Symmetry codes: (i) $x-\frac{1}{2},-y+\frac{1}{2},-z+1$; (ii) $-x, y+\frac{1}{2},-z+\frac{3}{2}$; (iii) $x, y+1, z$.

Table 2

Hydrogen-bond geometry $\left(\AA,^{\circ}\right)$ for (II).

$\operatorname{Cg} D$ and $\operatorname{Cg} E$ are the centroids of the pyridine and quinoline rings, respectively.

\begin{tabular}{lllll}
\hline$D-\mathrm{H} \cdots A$ & $D-\mathrm{H}$ & $\mathrm{H} \cdots A$ & $D \cdots A$ & $D-\mathrm{H} \cdots A$ \\
\hline $\mathrm{C} 32-\mathrm{H} 321 \cdots \mathrm{O} 2$ & 0.93 & 2.58 & $2.936(7)$ & 103 \\
$\mathrm{C} 22-\mathrm{H} 221 \cdots \mathrm{Cg} D^{\mathrm{i}}$ & 0.93 & 2.92 & $3.689(7)$ & 141 \\
$\mathrm{C} 11-\mathrm{H} 111 \cdots \mathrm{Cg} E^{\mathrm{ii}}$ & 0.93 & 2.92 & $3.681(7)$ & 140 \\
\hline
\end{tabular}

Symmetry codes: (i) $x+\frac{1}{2},-y+\frac{1}{2},-z+1$; (ii) $-x+1, y-\frac{1}{2},-z+\frac{3}{2}$.

\section{References}

Altomare, A., Cascarano, G., Giacovazzo, C. \& Guagliardi, A. (1993). J. Appl. Cryst. 26, 343-350.

Blessing, R. H. (1995). Acta Cryst. A51, 33-38.

Braje, W., Holzgrefe, J., Wartchow, R. \& Hoffman, H. M. R. (2000). Angew. Chem. Int. Ed. 39, 2085-2087.

Brunner, H. \& Bügler, J. (1997). Bull. Soc. Chim. Belg. 106, 77-84.

Bylaska, E. J. et al. (2006). NWChem. Version 5.0. Pacific Northwest National Laboratory, Richland, Washington, USA.

Caner, H., Biedermann, P. U. \& Agranat, I. (2003). Chirality, 15, 637-645.

Farrugia, L. J. (1997). J. Appl. Cryst. 30, 565.

Farrugia, L. J. (1999). J. Appl. Cryst. 32, 837-838.

Flack, H. D. (1983). Acta Cryst. A39, 876-881.

Gałdecki, Z., Kowalski, A., Kucharczyk, D. \& Uszyński, L. (1996). KM4B8. Kuma Diffraction, Wrocław, Poland.

Gałdecki, Z., Kowalski, A. \& Uszyński, L. (1995). DATAPROC. Version 9.0. Kuma Diffraction, Wrocław, Poland.

Hoffman, H. M. R. \& Frackenpohl, J. (2004). Eur. J. Org. Chem. pp. 42934312.

Hypercube (1998). HYPERCHEM. Release 4.5. Hypercube Inc., Waterloo, Ontario, Canada.

Jorgensen, W. L., Chandrasekhar, J., Madura, J. D., Impey, R. W. \& Klein, L. (1983). J. Chem. Phys. 79, 926-935.

Kendall, R. A., Apra, E., Bernhold, D. E., Bylaska, E. J., Dupois, M., Fann, G. I., Harrison, R. J., Ju, J., Nichols, J. A., Nieplocha, J., Straatsma, T. P., Windus, T. L. \& Wong, A. T. (2000). Comput. Phys. Commun. 128, 260283.

Kowalik, J. T., Lipińska, T. M., Oleksyn, B. \& Śliwiński, J. (1999). Enantiomer, 4, 389-410.

North, A. C. T., Phillips, D. C. \& Mathews, F. S. (1968). Acta Cryst. A24, 351359.

Oleksyn, B. J. (1982). Acta Cryst. B38, 1832-1834.

Oleksyn, B., Lebioda, Ł. \& Ciechanowicz-Rutkowska, M. (1979). Acta Cryst. B35, 440-444.

Sheldrick, G. M. (2008). Acta Cryst. A64, 112-122.

Song, E. C. (2009). In Cinchona Alkaloids in Synthesis and Catalysis. Weinheim: Wiley-VCH.

Verpoorte, R., Schripsema, J. \& Der Leer, T. V. (1988). The Alkaloids, Vol. 34, edited by A. Brossi, pp. 331-398. San Diego: Academic Press. 